\title{
Homoclinic orbits of sub-linear Hamiltonian systems with perturbed terms
}

\author{
Haiyan LV and Guanwei Chen ${ }^{2 *}$ (B)
}

"Correspondence:
guanweic@163.com
2School of Mathematical Sciences,
University of Jinan, Jinan 250022,
Shandong Province, P.R. China
Full list of author information is
available at the end of the article

available at the end of the article

\begin{abstract}
By using variational methods, we obtain the existence of homoclinic orbits for perturbed Hamiltonian systems with sub-linear terms. To the best of our knowledge, there is no published result focusing on the perturbed and sub-linear Hamiltonian systems.
\end{abstract}

MSC: $37 \mathrm{~J} 45 ; 37 \mathrm{~K} 05$

Keywords: Perturbed Hamiltonian systems; Sub-linear; Homoclinic orbits

\section{Introduction and the main result}

In this paper, we study the existence of homoclinic orbits for the following second order Hamiltonian systems with perturbed terms:

$$
-\ddot{u}(t)+A(t) u(t)-\lambda u(t)=\chi(t) \nabla F(u(t))+h(t), \quad t \in \mathbb{R},
$$

where $u \in \mathbb{R}^{N}, A(t)$ is continuous $T$-periodic $N \times N$ symmetric matrix valued function, $\lambda \in \mathbb{R}, h \in \mathbb{R}^{N}, F(u) \in C^{1}\left(\mathbb{R}^{N}, \mathbb{R}\right)$ and $\nabla F(u)$ denotes its gradient with respect to the $u$ variable.

As usual, we say that $u(t)$ is a homoclinic orbit of (1.1) if $u(t)$ is a solution of (1.1) and $u(t) \in C^{2}\left(\mathbb{R}, \mathbb{R}^{N}\right)$ such that $u(t) \rightarrow 0$ as $|t| \rightarrow \infty$.

If $\lambda=0$ and $h(t) \equiv 0$, then the system (1.1) becomes to the second order Hamiltonian system

$$
-\ddot{u}(t)+A(t) u(t)=\chi(t) \nabla F(u(t)), \quad t \in \mathbb{R} .
$$

Since homoclinic orbits play a key role in the research of fluid mechanics and gas dynamics. Therefore, homoclinic orbits of Hamiltonian systems have been studied by many authors $[1-12,14-21]$. If the matrix $A(t)$ is positive definite uniformly in $t$, the authors $[7-9,18,19]$ have obtained the existence of homoclinic orbits for (1.2). However, we shall consider the cases of (1.1) where $A(t)$ is not uniformly positively definite for $t \in \mathbb{R}$ and the nonlinearities $\nabla F(u)$ is sub-linear as at infinity.

In this paper, we are interested in the strongly indefinite case for (1.1).

(c) The Author(s) 2021. This article is licensed under a Creative Commons Attribution 4.0 International License, which permits use, sharing, adaptation, distribution and reproduction in any medium or format, as long as you give appropriate credit to the original author(s) and the source, provide a link to the Creative Commons licence, and indicate if changes were made. The images or other third party material in this article are included in the article's Creative Commons licence, unless indicated otherwise in a credit line to the material. If material is not included in the article's Creative Commons licence and your intended use is not permitted by statutory regulation or exceeds the permitted use, you will need to obtain permission directly from the copyright holder. To view a copy of this licence, visit http://creativecommons.org/licenses/by/4.0/. 
$\left(L_{1}\right) A(t)$ is $T$-periodic, $\lambda \notin \sigma\left(-\frac{d^{2}}{d t^{2}}+A(t)\right)$, the spectrum of $-\frac{d^{2}}{d t^{2}}+A(t)$.

Let $(\cdot, \cdot)$ be the inner product in $\mathbb{R}^{N}$, and the associated norm is denoted by $|\cdot|$. Assume that

$(X 1) h \in L^{2}\left(\mathbb{R}, \mathbb{R}^{N}\right), \chi \in L^{2}(\mathbb{R}, \mathbb{R}) \cap L^{\infty}(\mathbb{R}, \mathbb{R})$ and $\chi(t) \geq 0$ for all $t \in \mathbb{R}$.

(F1) $F(u) \in C^{1}\left(\mathbb{R}^{N}, \mathbb{R}\right), F(u) \geq 0$ for all $u \in \mathbb{R}^{N}, F(0)=0$ and

$$
\lim _{|u| \rightarrow \infty} \frac{|\nabla F(u)|}{|u|}=0 .
$$

By $F(0)=0$ and the differential mean value theorem, we have

$$
F(u)=F(u)-F(0)=(\nabla F(s u), u) \text { for some } s \in(0,1) .
$$

It follows from (1.3) that

$$
\lim _{|u| \rightarrow \infty} \frac{|F(u)|}{|u|^{2}}=\lim _{|u| \rightarrow \infty} \frac{|(\nabla F(s u), u)|}{|u|^{2}} \leq \lim _{|u| \rightarrow \infty} \frac{|\nabla F(s u)|}{|s u|}|s|=0,
$$

so we get $\lim _{|u| \rightarrow \infty} \frac{F(u)}{|u|^{2}}=0$, i.e., the nonlinearity $F(u)$ shows sub-quadratic growth at infinity. Now, our main result reads as follows.

\section{Theorem 1.1}

(1) If $F(0)=0$ and $h(t) \equiv 0$, then (1.1) has at least one homoclinic orbit.

(2) If $\left(L_{1}\right),(X 1)$ and $(F 1)$ hold, and $h(t) \not \equiv 0$, then (1.1) has at least one nontrivial homoclinic orbit.

Remark 1.1 To the best of our knowledge, there is no published result focusing on the perturbed Hamiltonian systems (1.1). The main novelties of this paper are as follows: (1) We extend the existing results of (1.2) to the more general Hamiltonian systems (1.1). (2) Most existing results of the systems (1.2) are based on the following superlinear conditions at 0 :

$$
|\nabla F(u)|=o(|u|) \quad \text { as }|u| \rightarrow 0, \quad \text { i.e., } \quad \lim _{|u| \rightarrow 0} \frac{|\nabla F(u)|}{|u|}=0 \text {. }
$$

However, we remove the conditions (1.4); (3) When the nonlinearity $\nabla F(u)$ is sub-linear as $|u| \rightarrow \infty$, as far as we know, the results on homoclinic orbits of (1.2) obtained in the literature are very scarce. Moreover, we obtain the existence of homoclinic orbits of the more general Hamiltonian systems (1.1) with $\nabla F(u)$ being sub-linear as $|u| \rightarrow \infty$.

\section{Variational frameworks and proof of the main result}

Let $E^{-}$be a separable closed subspace of a Hilbert space $E$ with inner product $\langle\cdot, \cdot\rangle$ and norm $\|\cdot\|$, and $E^{+}=\left(E^{-}\right)^{\perp}$. For some $R>0$, set

$$
M:=\left\{u \in E^{-}:\|u\| \leq R\right\}
$$

Then $M$ is a submanifold of $E^{-}$with boundary $\partial M$. On $E$ we will also use a topology $\tau$ generated by the norm

$$
\|u\|_{\tau}:=\max \left(\left\|P_{+} u\right\|, \sum_{k=1}^{\infty} \frac{1}{2^{k+1}}\left|\left\langle P_{-} u, e_{k}\right\rangle\right|\right)
$$


where $P_{ \pm}: E \rightarrow E^{ \pm}$is the orthogonal projection of $E$ onto $E^{ \pm}$and $\left\{e_{k}\right\}$ is a total orthonormal sequence in $E^{-}$. Obviously,

$$
u^{j} \stackrel{\tau}{\rightarrow} u \quad \text { implies that } \quad P_{+} u^{j} \rightarrow P_{+} u \quad \text { and } \quad P_{-} u^{j} \rightarrow P_{-} u .
$$

Notations We shall denote by $\|\cdot\|_{L^{q}}$ and $\|\cdot\|_{q}$ the usual $L^{q}\left(\mathbb{R}, \mathbb{R}^{N}\right)$-norm and $L^{q}(\mathbb{R}, \mathbb{R})$ norm $(1 \leq q \leq \infty)$, respectively. We denote by $C$ different positive constants. Let $\Phi \in$ $C^{1}(E, \mathbb{R})$. We say that $\Phi$ is $\tau$-upper semicontinuous if $u^{j} \stackrel{\tau}{\rightarrow} u$ implies $\Phi(u) \geq \overline{\lim }_{j \rightarrow \infty} \Phi\left(u^{j}\right)$, and $\Phi^{\prime}$ is weakly sequentially continuous if $u^{j} \rightarrow u$ implies $\Phi^{\prime}\left(u^{j}\right) \rightarrow \Phi^{\prime}(u)$.

Next, we shall use the following generalized saddle point theorem to prove our main result.

Lemma 2.1 ([13]) Suppose that $\Phi \in C^{1}(E, \mathbb{R})$ is $\tau$-upper semicontinuous and $\Phi^{\prime}$ is weakly sequentially continuous. If

$$
b:=\inf _{E^{+}} \Phi>\sup _{\partial M} \Phi, \quad d:=\sup _{M} \Phi<\infty,
$$

then, for some $c \in[b, d]$, there is $a(P S)_{c}$ sequence $\left\{u^{j}\right\} \subset E$, i.e., $\Phi\left(u^{j}\right) \rightarrow c$ and $\Phi^{\prime}\left(u^{j}\right) \rightarrow 0$.

Under assumption $\left(L_{1}\right), B:=-\frac{d^{2}}{d t^{2}}+A(t)-\lambda$ is a selfadjoint operator acting on $L^{2}:=$ $L^{2}\left(\mathbb{R}, \mathbb{R}^{N}\right)$ with domain $\mathcal{D}(B)=H^{2}\left(\mathbb{R}, \mathbb{R}^{N}\right)$ and we have the orthogonal decomposition $L^{2}=$ $L^{-} \oplus L^{+}, u=u^{-}+u^{+}$such that $B$ is negative (resp., positive) in $L^{-}$(resp., in $L^{+}$). Let $E:=$ $\mathcal{D}\left(|B|^{1 / 2}\right)$ be equipped, respectively, with the inner product and norm

$$
\langle u, v\rangle:=\left(|B|^{1 / 2} u,|B|^{1 / 2} v\right)_{L^{2}}, \quad\|u\|:=\left\||B|^{1 / 2} u\right\|_{L^{2}},
$$

where $(\cdot, \cdot)_{L^{2}}$ denotes the inner product of $L^{2}\left(\mathbb{R}, \mathbb{R}^{N}\right)$. Then we have the decomposition

$$
E=E^{-} \oplus E^{+}
$$

and

$$
E^{ \pm}=E \cap L^{ \pm}
$$

orthogonal with respect to both $(\cdot, \cdot)_{L^{2}}$ and $\langle\cdot, \cdot\rangle$. By $\left(L_{1}\right), E=H^{1}\left(\mathbb{R}, \mathbb{R}^{N}\right)$ with equivalent norms. Then $E$ is a Hilbert space and it is not difficult to show that $E \subset C^{0}\left(\mathbb{R}, \mathbb{R}^{N}\right)$, the space of continuous functions $u$ on $\mathbb{R}$ such that $u(t) \rightarrow 0$ as $|t| \rightarrow \infty$ (see, e.g., [18]).

Therefore, the corresponding functional of (1.1) can be written as

$$
\begin{aligned}
\Phi(u) & =\frac{1}{2} \int_{\mathbb{R}}\left[|\dot{u}|^{2}+(A(t) u, u)-\lambda u^{2}\right] d t-\Psi(u) \\
& =\frac{1}{2}\left(\left\|u^{+}\right\|^{2}-\left\|u^{-}\right\|^{2}\right)-\Psi(u), \quad u \in E,
\end{aligned}
$$

where $\Psi(u):=\int_{\mathbb{R}}[\chi(t) F(u)+h(t) u] d t$. By assumptions $\left(L_{1}\right),(X 1)$ and $(F 1)$, it is easy to verify that $\Phi, \Psi \in C^{1}(E, \mathbb{R})$ and the derivatives are given by

$$
\Phi^{\prime}(u) v=\left\langle u^{+}, v^{+}\right\rangle-\left\langle u^{-}, v^{-}\right\rangle-\Psi^{\prime}(u) v, \quad \Psi^{\prime}(u) v=\int_{\mathbb{R}}[\chi(t)(\nabla F(u), v)+h(t) v] d t,
$$


where $u=u^{-}+u^{+}, v=v^{-}+v^{+} \in E=E^{-} \oplus E^{+}$. Equation (2.4) implies that (1.1) is the corresponding Euler-Lagrange equation for $\Phi$. Therefore, we have reduced the problem of finding homoclinic orbits of (1.1) to that of seeking critical points of the functional $\Phi$ on $E$. In order to apply Lemma 2.1 to prove our result, we need the following two lemmas.

Lemma 2.2 Under conditions of Theorem 1.1, the functional $\Phi$ is $\tau$-upper semicontinuous and $\Phi^{\prime}$ is weakly sequentially continuous.

Proof First, we show that the functional $\Phi$ is $\tau$-upper semicontinuous. Let $u^{j} \stackrel{\tau}{\rightarrow} u$ and $\Phi\left(u^{j}\right) \geq C_{0}$ for some constant $C_{0}$. By (2.2), we have

$$
\left(u^{j}\right)^{+} \rightarrow u^{+}, \quad\left(u^{j}\right)^{-} \rightarrow u^{-} \quad \text { and } \quad u^{j} \rightarrow u \quad \text { in } E, \quad u^{j} \rightarrow u \quad \text { a.e. on } \mathbb{R}^{N},
$$

going to a subsequence if necessary. Clearly, $(X 1)$ and $(F 1)$ imply $\chi(t) F(u) \geq 0$ for all $(t, u) \in$ $\mathbb{R} \times \mathbb{R}^{N}$, which together with (2.5) and Fatou's lemma implies

$$
\varliminf_{j \rightarrow \infty} \int_{\mathbb{R}} \chi(t) F\left(u^{j}\right) d t \geq \int_{\mathbb{R}} \chi(t) F(u) d t .
$$

By (2.5), we have $u^{j} \rightarrow u$ in $L^{2}\left(\mathbb{R}, \mathbb{R}^{N}\right)$, it follows from $h \in L^{2}\left(\mathbb{R}, \mathbb{R}^{N}\right)$ (see $\left.(X 1)\right)$ that

$$
\lim _{j \rightarrow \infty} \int_{\mathbb{R}} h(t) u^{j} d t=\lim _{j \rightarrow \infty}\left(h, u^{j}\right)=(h, u)=\int_{\mathbb{R}} h(t) u d t .
$$

By (2.6), (2.7), $\Phi\left(u^{j}\right) \geq C_{0}$, the definition of $\Phi$ and the weak lower semicontinuity of the norm, we get

$$
\begin{aligned}
-C_{0} & \geq \lim _{j \rightarrow \infty}\left(-\Phi\left(u^{j}\right)\right) \\
& =\lim _{j \rightarrow \infty} \frac{1}{2}\left(\left\|\left(u^{j}\right)^{-}\right\|^{2}-\left\|\left(u^{j}\right)^{+}\right\|^{2}\right)+\int_{\mathbb{R}}\left(\chi(t) F\left(u^{j}\right)+h(t) u^{j}\right) d t \\
& \geq \frac{1}{2}\left(\left\|u^{-}\right\|^{2}-\left\|u^{+}\right\|^{2}\right)+\int_{\mathbb{R}}(\chi(t) F(u)+h(t) u) d t=-\Phi(u) .
\end{aligned}
$$

It implies that $\Phi(u) \geq C_{0}$. Therefore, $\Phi$ is $\tau$-upper semicontinuous.

Now, we prove $\Phi^{\prime}$ is weakly sequentially continuous on $E$. By (2.5) and the definition of $\Phi^{\prime}$, we have

$$
\begin{aligned}
& \lim _{j \rightarrow \infty} \Phi^{\prime}\left(u^{j}\right) \varphi \\
& \quad=\lim _{j \rightarrow \infty}\left\{\left\langle\left(u^{j}\right)^{+}, \varphi\right\rangle-\left\langle\left(u^{j}\right)^{-}, \varphi\right\rangle-\int_{\mathbb{R}}\left[\chi(t)\left(\nabla F\left(u^{j}\right), \varphi\right)+h(t) \varphi\right] d t\right\} \\
& \quad=\left\langle u^{+}, \varphi\right\rangle-\left\langle u^{-}, \varphi\right\rangle-\lim _{j \rightarrow \infty} \int_{\mathbb{R}}\left[\chi(t)\left(\nabla F\left(u^{j}\right), \varphi\right)+h(t) \varphi\right] d t, \quad \forall \varphi \in C_{0}^{\infty}\left(\mathbb{R}, \mathbb{R}^{N}\right) .
\end{aligned}
$$

It follows from $F \in C^{1}, \varphi \in C_{0}^{\infty}\left(\mathbb{R}, \mathbb{R}^{N}\right)$ and $u^{j} \rightarrow u$ in $L_{\text {loc }}^{2}\left(\mathbb{R}, \mathbb{R}^{N}\right)$ (by (2.5)) that

$$
\lim _{j \rightarrow \infty} \Phi^{\prime}\left(u^{j}\right) \varphi=\left\langle u^{+}, \varphi\right\rangle-\left\langle u^{-}, \varphi\right\rangle-\int_{\mathbb{R}}[\chi(t)(\nabla F(u), \varphi)+h(t) \varphi] d t=\Phi^{\prime}(u) \varphi,
$$

i.e., $\Phi^{\prime}$ is weakly sequentially continuous on $E$. The proof is finished. 
Lemma 2.3 Under conditions of Theorem 1.1, the geometric assumption (2.3) in Lemma

2.1 is true, i.e.,

$$
b:=\inf _{E^{+}} \Phi>\sup _{\partial M} \Phi, \quad d:=\sup _{M} \Phi<\infty .
$$

Proof Obviously, if $\chi(t) \equiv 0(t \in \mathbb{R})$, then assumption $\left(L_{1}\right)$ implies that (1.1) becomes to a linear equation and it is easy to see that it has a solution. Therefore, we may assume that $\|\chi\|_{\infty} \neq 0$. By the Sobolev inequality, there is a constant $C_{0}>0$ such that

$$
C_{0}\|u\|_{L^{2}}^{2} \leq\|u\|^{2}, \quad \forall u \in E
$$

Clearly, (F1) implies that

$$
|\nabla F(u)| \leq \frac{C_{0}}{3\|\chi\|_{\infty}}|u|+C, \quad|F(u)| \leq \frac{C_{0}}{3\|\chi\|_{\infty}}|u|^{2}+C|u|, \quad u \in \mathbb{R}^{N} .
$$

For $u \in E^{+}$, by (2.8), (2.9), the definition of $\Phi$ and the Hölder inequality, we have

$$
\begin{aligned}
\Phi(u) & =\frac{1}{2}\|u\|^{2}-\int_{\mathbb{R}}(\chi(t) F(u)+h(t) u) d t \\
& \geq \frac{1}{2}\|u\|^{2}-\int_{\mathbb{R}}|\chi(t)|\left(\frac{C_{0}}{3\|\chi\|_{\infty}}|u|^{2}+C|u|\right) d t-\int_{\mathbb{R}}|h(t)||u| d t \\
& \geq \frac{1}{2}\|u\|^{2}-\frac{C_{0}}{3}\|u\|_{L^{2}}^{2}-\left(C\left\|_{\chi}\right\|_{2}+\|h\|_{L^{2}}\right)\|u\|_{L^{2}} \\
& \geq \frac{1}{6}\|u\|^{2}-\frac{1}{C_{0}^{1 / 2}}\left(C\|\|_{2}+\|h\|_{L^{2}}\right)\|u\| .
\end{aligned}
$$

It follows from $\|\chi\|_{2}+\|h\|_{L^{2}}<\infty(\operatorname{see}(X 1))$ that $b:=\inf _{E^{+}} \Phi>-\infty$

For $u \in E^{-}$, by (2.8) and $\chi(t) F(u) \geq 0$ for all $(t, u) \in \mathbb{R} \times \mathbb{R}^{N}$ (see $(X 1)$ and $(F 1)$ ), we have

$$
\begin{aligned}
\Phi(u) & =-\frac{1}{2}\|u\|^{2}-\int_{\mathbb{R}}\left(\chi_{n} F_{n}\left(u_{n}\right)+h_{n} u_{n}\right) d t \\
& \leq-\frac{1}{2}\|u\|^{2}+\|h\|_{L^{2}}\|u\|_{L^{2}} \\
& \leq-\frac{1}{2}\|u\|^{2}+\frac{1}{C_{0}^{1 / 2}}\|h\|_{L^{2}}\|u\| .
\end{aligned}
$$

It follows from $\|h\|_{L^{2}}<\infty$ (see $\left.(X 1)\right)$ that for $R$ large enough we have

$$
b:=\inf _{E^{+}} \Phi>\sup _{\partial M} \Phi, \quad d:=\sup _{M} \Phi<\infty,
$$

where $M$ is defined in (2.1). The proof is finished.

Now, Lemmas 2.2 and 2.3 imply that Lemma 2.1 holds. Next, we give a detailed proof of Theorem 1.1.

Proofs of Theorem 1.1(1) and (2) (1) Obviously, 0 is a trivial homoclinic orbit of (1.1) if $F(0)=0$ and $h(t) \equiv 0$. 
(2) By Lemma 2.1, for some $c \in[b, d]$, there is a sequence $\left\{u^{j}\right\} \subset E$ such that

$$
\Phi\left(u^{j}\right) \rightarrow c, \quad \Phi^{\prime}\left(u^{j}\right) \rightarrow 0
$$

Let $\hat{u}^{j}=\left(u^{j}\right)^{+}-\left(u^{j}\right)^{-}$, then $\left\|\hat{u}^{j}\right\|=\left\|u^{j}\right\|$. Therefore, by (2.8)-(2.10), the Hölder inequality and the fact $E=E^{-} \oplus E^{+}$orthogonal with respect to $(\cdot, \cdot)_{L^{2}}$, we have

$$
\begin{aligned}
C\left\|u^{j}\right\| & =C\left\|\hat{u}^{j}\right\| \geq \Phi^{\prime}\left(u^{j}\right) \hat{u}^{j} \\
& =\left\|\left(u^{j}\right)^{+}\right\|^{2}+\left\|\left(u^{j}\right)^{-}\right\|^{2}-\int_{\mathbb{R}}\left[\chi(t)\left(\nabla F\left(u^{j}\right), \hat{u}^{j}\right)+h(t) \hat{u}^{j}\right] d t \\
& \geq\left\|u^{j}\right\|^{2}-\int_{\mathbb{R}}\left[|\chi(t)|\left(\frac{C_{0}}{3\|\chi\|_{\infty}}\left|u^{j}\right|+C\right)+|h(t)|\right]\left(\left|\left(u^{j}\right)^{+}\right|+\left|\left(u^{j}\right)^{-}\right|\right) d t \\
& \geq\left\|u^{j}\right\|^{2}-\left(\frac{C_{0}}{3}\left\|u^{j}\right\|_{L^{2}}+C\left\|_{\chi}\right\|_{2}+\|h\|_{L^{2}}\right)\left(\left\|\left(u^{j}\right)^{+}\right\|_{L^{2}}+\left\|\left(u^{j}\right)^{-}\right\|_{L^{2}}\right) \\
& \geq\left\|u^{j}\right\|^{2}-2\left\|u^{j}\right\|_{L^{2}}\left(\frac{C_{0}}{3}\left\|u^{j}\right\|_{L^{2}}+C\|\chi\|_{2}+\|h\|_{L^{2}}\right) \\
& \geq \frac{1}{3}\left\|u^{j}\right\|^{2}-\frac{2}{C_{0}^{1 / 2}}\left(C\|\chi\|_{2}+\|h\|_{L^{2}}\right)\left\|u^{j}\right\| .
\end{aligned}
$$

It follows from $\|\chi\|_{2}+\|h\|_{L^{2}}<\infty$ (see $\left.(X 1)\right)$ that $\left\{u^{j}\right\}$ is bounded in $E$.

Consequently, up to a subsequence, we may assume that $u^{j} \rightarrow u$ in $E$. By (2.10) and the fact that $\Phi^{\prime}$ is weakly sequentially continuous (see Lemma 2.2), we have

$$
0=\lim _{j \rightarrow \infty} \Phi^{\prime}\left(u^{j}\right) v=\Phi^{\prime}(u) v, \quad \forall v \in E
$$

Therefore, $u$ is a homoclinic orbit of (1.1). The fact $h(t) \not \equiv 0$ implies the system (1.1) has no trivial solution, i.e., 0 is not a solution of (1.1), thus $u$ is a nontrivial homoclinic orbit of (1.1). The proof is finished.

\section{Conclusion}

We obtain the existence of homoclinic orbits for a class of perturbed Hamiltonian systems with sub-linear terms. To the best of our knowledge, there is no published result focusing on the perturbed and sub-linear Hamiltonian systems.

Acknowledgements

We would like to thank the editors for their valuable comments and suggestions, which have led to an improvement of the presentation of this paper.

\section{Funding}

Research supported by National Natural Science Foundation of China (No. 11771182, No. 11801012) and the Foundation for Excellent Youth Teachers of Colleges and Universities of Henan Province (No. 2020GGJS189).

\section{Abbreviations}

Not applicable.

Availability of data and materials Not applicable.

Ethics approval and consent to participate 


\section{Competing interests}

The authors declare that they have no competing interests.

\section{Consent for publication}

Not applicable.

\section{Authors' contributions}

All authors contributed equally to the writing of this paper. All authors read and approved the final manuscript.

\section{Author details}

${ }^{1}$ School of Mathematics and Statistics, Anyang Normal University, Anyang 455000, Henan Province, P.R. China. ${ }^{2}$ School of Mathematical Sciences, University of Jinan, Jinan 250022, Shandong Province, P.R. China.

\section{Publisher's Note}

Springer Nature remains neutral with regard to jurisdictional claims in published maps and institutional affiliations.

Received: 4 January 2021 Accepted: 14 March 2021 Published online: 21 March 2021

\section{References}

1. Alves, C.O., Carrião, P.C., Miyagaki, O.H.: Existence of homoclinic orbits for asymptotically periodic systems involving Duffing-like equation. Appl. Math. Lett. 16(5), 639-642 (2003)

2. Ambrosetti, A., Rabinowitz, P.H.: Dual variational methods in critical point theory and applications. J. Funct. Anal. 14(4), 349-381 (1973)

3. Carrião, P.C., Miyagaki, O.H.: Existence of homoclinic solutions for a class of time-dependent Hamiltonian systems. J. Math. Anal. Appl. 230(1), 157-172 (1999)

4. Chen, G.: Homoclinic orbits for second order Hamiltonian systems with asymptotically linear terms at infinity. Adv. Differ. Equ. 2014, 114 (2014)

5. Chen, G.: Superquadratic or asymptotically quadratic Hamiltonian systems: ground state homoclinic orbits. Ann. Mat. Pura Appl. 194, 903-918 (2015)

6. Chen, G.: Homoclinic orbits of first order nonlinear Hamiltonian systems with asymptotically linear nonlinearities at infinity. Topol. Methods Nonlinear Anal. 47, 499-510 (2016)

7. Coti Zelati, V., Rabinowitz, P.H.: Homoclinic orbits for second order Hamiltonian systems possessing superquadratic potentials. J. Am. Math. Soc. 4(4), 693-727 (1991)

8. Ding, Y., Girardi, M.: Periodic and homoclinic solutions to a class of Hamiltonian systems with the potentials changing sign. Dyn. Syst. Appl. 2(1), 131-145 (1993)

9. Ding, Y., Lee, C.: Homoclinics for asymptotically quadratic and superquadratic Hamiltonian systems. Nonlinear Anal. 71, 1395-1413 (2009)

10. Flavia, A.: Periodic and homoclinic solutions to a class of Hamiltonian systems with indefinite potential in sign. Boll. Unione Mat. Ital., B (7) 10(2), 303-324 (1996)

11. Izydorek, M., Janczewska, J.: Homoclinic solutions for a class of the second order Hamiltonian systems. J. Differ. Equ. 219(2), 375-389 (2005)

12. Korman, P., Lazer, A.C.: Homoclinic orbits for a class of symmetric Hamiltonian systems. Electron. J. Differ. Equ. 1994, 01 (1994)

13. Liu, S., Shen, Z:: Generalized saddle point theorem and asymptotically linear problems with periodic potential. Nonlinear Anal. 86, 52-57 (2013)

14. Liu, Z., Guo, S., Zhang, Z.: Homoclinic orbits for the second-order Hamiltonian systems. Nonlinear Anal., Real World Appl. 36, 116-138 (2017)

15. LV, X.: Homoclinic solutions for a class of second-order Hamiltonian systems with locally defined potentials. Electron. J. Differ. Equ. 2017, 205 (2017)

16. Omana, W., Willem, M.: Homoclinic orbits for a class of Hamiltonian systems. Differ. Integral Equ. 5(5), 1115-1120 (1992)

17. Paturel, E.: Multiple homoclinic orbits for a class of Hamiltonian systems. Calc. Var. Partial Differ. Equ. 12(2), 117-143 (2001)

18. Rabinowitz, P.H.: Homoclinic orbits for a class of Hamiltonian systems. Proc. R. Soc. Edinb., Sect. A 114, 33-38 (1990)

19. Rabinowitz, P.H., Tanaka, K.: Some results on connecting orbits for a class of Hamiltonian systems. Math. Z. 206(3), 473-499 (1991)

20. Wang, J., Zhang, F., Xu, J.: Existence and multiplicity of homoclinic orbits for the second order Hamiltonian systems. J. Math. Anal. Appl. 366, 569-581 (2010)

21. Zou, W., Li, S.: Infinitely many homoclinic orbits for the second-order Hamiltonian systems. Appl. Math. Lett. 16 $1283-1287$ (2003) 